\title{
The Low Temperature Specific Heat of $\operatorname{Pr}_{0.65} \mathrm{Ca}_{0.35} \mathrm{MnO}_{3}$
}

\author{
Zhiyong Han and Zhenzhu Jing \\ Tianjin Key Laboratory of Civil Aircraft Airworthiness a Maintenance, Civil Aviation University of China, Tianjin 300300, China \\ Correspondence should be addressed to Zhiyong Han; zyhan@cauc.edu.cn
}

Received 10 March 2014; Revised 4 July 2014; Accepted 10 August 2014; Published 25 August 2014

Academic Editor: Ashok Chatterjee

Copyright (C) 2014 Z. Han and Z. Jing. This is an open access article distributed under the Creative Commons Attribution License, which permits unrestricted use, distribution, and reproduction in any medium, provided the original work is properly cited.

\begin{abstract}
The low temperature specific heat of polycrystalline perovskite-type $\operatorname{Pr}_{0.65} \mathrm{Ca}_{0.35} \mathrm{MnO}_{3}$ manganese oxide has been investigated experimentally. It is found that the low temperature electron specific heat in zero magnetic field is obviously larger than that of ordinary rare-earth manganites oxide. To get the contribution of phonon to the specific heat precisely, the lattice specific heat is calculated by Debye model fitting. Results confirm that the low temperature specific heat of $\operatorname{Pr}_{0.65} \mathrm{Ca}_{0.35} \mathrm{MnO}_{3}$ is related to the itinerant electrons in ferromagnetic clusters and the disorder in the sample.
\end{abstract}

\section{Introduction}

Mix-valent perovskite manganese oxide $\mathrm{R}_{1-x} \mathrm{~A}_{x} \mathrm{MnO}_{3}$ (where $\mathrm{R}$ and $\mathrm{A}$ stand for rare-earth and alkaline-earth metal elements, resp.) is regarded as a typical material of strongly correlated system with strong interplay between electric charge, spin, orbit, and crystal lattice degrees of freedom controlling the electromagnetic properties of this kind of oxide $[1,2]$. By studying the electric charge orbit and spin of the perovskite manganese oxide, it is helpful to understand the physical mechanisms of the change from the insulator to metal and colossal magnetoresistance (CMR) effect. However, due to the limitation of the low temperature technology, it is hard to observe these factors by experiment directly. The low temperature specific heat research is an effective method to understand the low temperature physical property of this kind of oxide.

The specific heat is a characteristic of the material itself. It is sensitive to many factors, such as the lattice thermal movement (the phonon specific heat) and the electron thermal movement (the electronic specific heat). When the specific heat of strongly correlated system is researched, the electronic energy state density around the Fermi energy can be known through the electronic specific heat. And it is closely related with materials electrical transport properties. Apart from the contribution of the lattice and the electron to the specific heat, the hyperfine structure and spin wave should be considered generally. Woodfield et al. [3] analyzed the specific heat of $\mathrm{La}_{1-x} \mathrm{Sr}_{x} \mathrm{MnO}_{3}$ and got the impact of each term on $x$ and the specific heat. Lees et al. [4] studied the low temperature specific heat of $\operatorname{Pr}_{0.6}\left(\mathrm{Ca}_{1-x} \mathrm{Sr}_{x}\right) \mathrm{MnO}_{3}$ with the same analysis technique as Woodfield et al. and the research results indicated that the electronic specific heat can reflect on the sample conductivity and the electronic energy state around the Fermi energy to a certain extent. Research on the electronic energy state density around the Fermi energy with the low temperature specific heat, therefore, has attracted great attention for a long time.

In this paper, contributions of the electron thermal movement, spin wave, and lattice thermal movement in different magnetization filed can be investigated by measuring the $\mathrm{Pr}_{0.65} \mathrm{Ca}_{0.35} \mathrm{MnO}_{3}$ low temperature specific heat. Without applied magnetic field, $\operatorname{Pr}_{0.65} \mathrm{Ca}_{0.35} \mathrm{MnO}_{3}$ is found to have a huge electronic specific heat. And in the traditional low temperature antiferromagnetic state, it is found that the specific heat of this material is affected not only by the antiferromagnetic spin wave but also by the ferromagnetic spin wave.

\section{Experiment}

Polycrystalline $\operatorname{Pr}_{0.65} \mathrm{Ca}_{0.35} \mathrm{MnO}_{3}$ powder prepared by solgel method [5] is pressed into discs and sintered at $1373 \mathrm{~K}$ for 24 hours and then furnace cooled to room temperature. Thus, polycrystalline $\operatorname{Pr}_{0.65} \mathrm{Ca}_{0.35} \mathrm{MnO}_{3}$ sample can be obtained from the experiment above. The structure of sample 
TABLE 1: Parameters under different magnetic fields fitting to (2).

\begin{tabular}{|c|c|c|c|c|}
\hline $\mathrm{H} / \mathrm{kOe}$ & $\gamma / \mathrm{mJ} \cdot \mathrm{mol}^{-1} \cdot \mathrm{K}^{-2}$ & $\delta_{2} / \mathrm{mJ} \cdot \mathrm{mol}^{-1} \cdot \mathrm{K}^{-3}$ & $\delta_{3 / 2} / \mathrm{mJ} \cdot \mathrm{mol}^{-1} \cdot \mathrm{K}^{-5 / 2}$ & $\theta_{D} / \mathrm{K}$ \\
\hline 0 & 15.0 & 10.5 & 4.0 & 435 \\
\hline 30 & 0 & 9.0 & 13.0 & 389 \\
\hline 50 & 3.9 & 5.9 & 12.0 & 237 \\
\hline 70 & 5.8 & 0 & 12.5 & 161 \\
\hline 90 & 5.5 & 0 & 12.0 & 161 \\
\hline
\end{tabular}

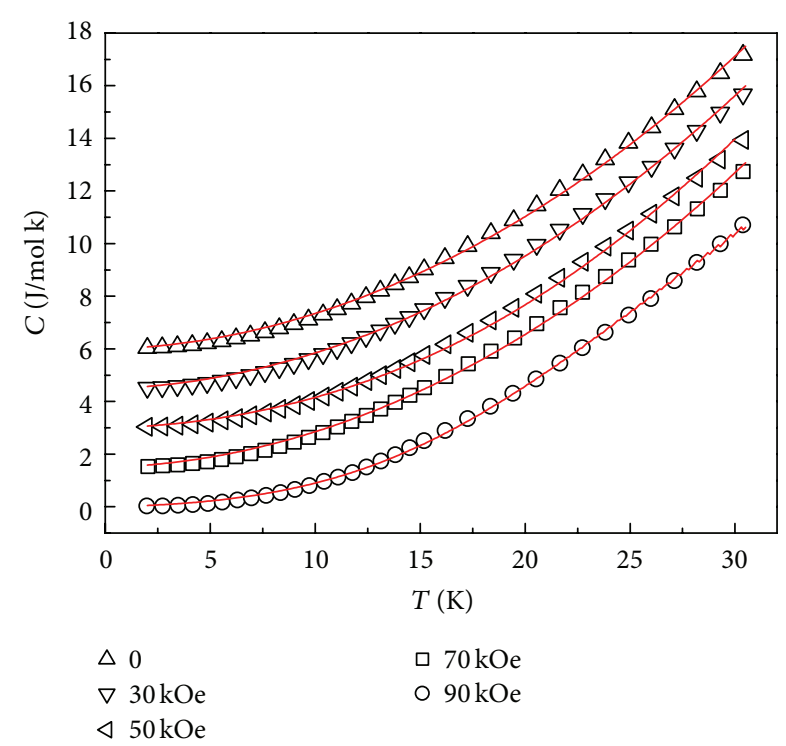

FIgURE 1: The specific heat versus temperature curve of polycrystalline $\operatorname{Pr}_{0.65} \mathrm{Ca}_{0.35} \mathrm{MnO}_{3}$ at low temperature under different magnetic fields. Solid line is fit to the data with (2).

is measured by $\mathrm{X}$-ray diffraction (XRD). The XRD results confirm the single phase with orthogonal structure and values of the lattice constants $a, b$, and $c$ are $5.4491 \AA, 5.4345 \AA$, and $7.6506 \AA$, respectively. The specific heat is measured through a physical property measurement system (PPMS) produced by Quantum Design company. The specific heat measurements of the sample are performed in magnetic field $(0,30 \mathrm{kOe}$, $50 \mathrm{kOe}, 70 \mathrm{kOe}$, and $90 \mathrm{kOe}$ ) and the temperature range is set as $2 \mathrm{~K}$ to $30 \mathrm{~K}$.

\section{Results and Discussion}

Low temperature $(2 \mathrm{~K}$ to $30 \mathrm{~K}$ ) specific heat of polycrystalline $\operatorname{Pr}_{0.65} \mathrm{Ca}_{0.35} \mathrm{MnO}_{3}$ changing with temperature is shown in Figure 1.

Generally, the low temperature specific heat can be calculated by the equation

$$
C(T)=\gamma T+\beta_{3} T^{3}+\beta_{5} T^{5}+\delta T^{n}+\frac{\alpha}{T^{2}},
$$

where $\gamma T$ is the electronic specific heat, $\beta_{3} T^{3}+\beta_{5} T^{5}$ is the phonon specific heat, $\delta T^{n}$ is the spin wave specific heat ( $n=3 / 2$ for ferromagnetic spin wave; $n=2$ for antiferromagnetic spin wave), and $\alpha / T^{2}$ is the specific heat of the hyperfine structure [4]. To analyze the contribution of phonon to the specific heat more precisely, the lattice specific heat is described by Debye model, with which results would be more reliable than using the high order term $\beta_{5} T^{5}$. Besides, taking into consideration both the ferromagnetic spin term $\left(T^{3 / 2}\right)$ and antiferromagnetic spin term $\left(T^{2}\right)$ at the same time, if the sample has one of them, the other spin structure would contribute nothing to specific heat; if both spin structures exist, they would contribute to the total specific heat simultaneously. Thus, the analysis equation could be written as

$$
\begin{aligned}
C(T)= & \frac{\alpha}{T^{2}}+\gamma T+\delta_{3 / 2} T^{3 / 2}+\delta_{2} T^{2} \\
& +9 R\left(\frac{T}{\theta_{D}}\right)^{3} \int_{0}^{T / \theta_{D}} \frac{\xi^{4} e^{\xi}}{\left(e^{\xi}-1\right)^{2}} d \xi .
\end{aligned}
$$

Here, the last term on the right-hand side represents Debye model specific heat, $R$ is the universal gas constant, and $\theta_{D}$ is the Debye temperature. In terms of least square method, the data in Figure 1 was fitted by (2), and the fitting parameters are shown in Table 1.

Reményi et al. [6] and Doerr et al. [7] had measured the magnetostriction of polycrystalline $\operatorname{Pr}_{0.65} \mathrm{Ca}_{0.35} \mathrm{MnO}_{3}$ and found that the longitudinal length shorts obviously as the magnetic field increases at low temperature $(T=45 \mathrm{~K}$ and $T=20 \mathrm{~K}$ ) and results in the decrease of lattice constant and lattice volume accordingly. The experimental results of this paper show that Debye temperature of $\operatorname{Pr}_{0.65} \mathrm{Ca}_{0.35} \mathrm{MnO}_{3}$ decreases with increasing magnetic field. That indicates a close relationship between thermal movements of phonon with lattice structure. As Debye temperature is proportional to the velocity of sound, our experimental results show that elasticity modulus of the material decreases with decreasing magnetic field, which is coordinated with magnetostriction experimental results of Remenyi and Doer's.

In our experiment, it is found that electronic specific coefficient $\gamma$ reached $15.0 \mathrm{~mJ} \cdot \mathrm{mol}^{-1} \cdot \mathrm{K}^{-2}$ without applied magnetic field, which contradicts with the previous value of macroconductivity. The value of $\gamma$ is related to electronic energy state density around the Fermi energy $\gamma=\left(\pi^{2} / 3\right) k_{B}^{2} N\left(E_{F}\right)$ and in the range of $3-7 \mathrm{~mJ} \cdot \mathrm{mol}^{-1} \cdot \mathrm{K}^{-2}$ for general metallic manganese $[3,4,8]$. For the metallic conductive manganese $\mathrm{La}_{1-x} \mathrm{Sr}_{x} \mathrm{MnO}_{3}, \gamma$ values are $0,3.3386 \mathrm{~mJ} \cdot \mathrm{mol}^{-1} \cdot \mathrm{K}^{-2}$ and $3.2873 \mathrm{~mJ} \cdot \mathrm{mol}^{-1} \cdot \mathrm{K}^{-2}$ when $x=0,0.1, x=0.2$, and $x=$ 0.3 , respectively. The electronic energy state density around the Fermi energy is $N\left(E_{F}\right)=2.4 \times 10^{22} \mathrm{eV}^{-1} \cdot \mathrm{cm}^{-3}$ which is very close to $N\left(E_{F}\right)=1.4 \times 10^{22} \mathrm{eV}^{-1} \cdot \mathrm{cm}^{-3}$ calculated 
through energy structure. Thus, $\gamma$ could directly reflect the electric structure around the Fermi energy [3]. It is found that in huge magnetic field of 50,70, and $90 \mathrm{kOe}$, values of $\gamma$ are $3.9,5.8$, and $5.5 \mathrm{~mJ} \cdot \mathrm{mol}^{-1} \cdot \mathrm{K}^{-2}$ respectively, which is very close to the general ferromagnetic metallic manganese. The phenomenon above shows that with the melting of $\mathrm{CO}$ in huge magnetic field, the material converts the $\mathrm{CO}$ insulator to ferromagnetic metallic state. People have found large $\gamma$ values in the insulator perovskite manganese [9-13], especially that Woodfield et al. found the electronic specific heat $\gamma=$ $15.7 \mathrm{~mJ} \cdot \mathrm{mol}^{-1} \cdot \mathrm{K}^{-2}$ in the polycrystalline $\mathrm{Pr}_{0.65} \mathrm{Ca}_{0.35} \mathrm{MnO}_{3}$ [3]. And large $\gamma$ means large electronic energy state density around the Fermi energy.

For $\mathrm{Pr}_{1-x} \mathrm{Ca}_{x} \mathrm{MnO}_{3}$, the low temperature magnetic order is the spin reorientation pseudo-CE structure when $x<$ 0.5 . In this metastable structure, it is probable that, due to the partial magnetic moment deviation, spin ranks parallelly and leads to partial ferromagnetic spin structure. In the meanwhile, the competence between the ferromagnetic and antiferromagnetic could result in the forming of cluster glass. Magnetic measurement results above confirm the existence of cluster glass in the sample. When the size of cluster is large enough, the electrons could be itinerant according to DE mode. But the different magnetic directions and distance between clusters hinder the electronic exchange of clusters, which could not form the long-range magnetic order structure. Though not forming the metallic electron conductivity, the cluster electron thermal movement might contribute to the specific heat. Daoud-Aladine used the neutron diffraction method to study the spin structure of single crystal $\mathrm{Pr}_{0.6} \mathrm{Ca}_{0.4} \mathrm{MnO}_{3}$ and found that the sample at low temperature is not purely electronic order antiferromagnetic spin structure and electron is stuck in a pair of $\mathrm{Mn}$ ions for ferromagnetic double exchange effect and the socalled Zener polaron formed [14]. As for magnetic impurity particles, the Zener polarons exist in the antiferromagnetic phase and could lead to cluster glass. With the change of internal disorder in the sample and the damage of the periodic potential field by the ferromagnetic polaron, the Fermi energy might move to the energy band gap formed by the localized $e_{g}$ electron which is produced by the magnetic polaron. Even though there exists the electronic energy state around the Fermi energy which could give rise to the unusual electronic specific heat in the experiment, these localized electrons will not contribute to the electron conductivity. With increasing magnetic field, the sample converts to total ferromagnetic metallic state at low temperature and with the vanish of the mix-magnetic disorder, the contribution of antiferromagnetic spin wave vanishes gradually and the usual electronic specific heat turned to the normal ferromagnetic metallic state.

\section{Conclusions}

Through the study of low temperature specific heat of the polycrystalline $\operatorname{Pr}_{0.65} \mathrm{Ca}_{0.35} \mathrm{MnO}_{3}$, it is found that in huge magnetic field of 50,70, and $90 \mathrm{kOe}$, values of electronic specific coefficient $\gamma$ are $3.9,5.8 \mathrm{~m}$ and $5.5 \mathrm{~mJ} \cdot \mathrm{mol}^{-1} \cdot \mathrm{K}^{-2}$, respectively, which is very close to the general ferromagnetic metallic manganese. This phenomenon shows that, with the melting of $\mathrm{CO}$ in huge magnetic field, the material converts the $\mathrm{CO}$ insulator to ferromagnetic metallic state. Besides, it is found that the electronic specific heat $\gamma$ at low temperature $\mathrm{CO}$ insulator reaches $15.0 \mathrm{~mJ} \cdot \mathrm{mol}^{-1} \cdot \mathrm{K}^{-2}$ and the main reason for this unusual electronic specific heat might be itinerant electrons in the ferromagnetic cluster or the disorder phenomena of the sample.

\section{Conflict of Interests}

The authors declare that there is no conflict of interests regarding the publication of this paper.

\section{Acknowledgment}

The funding from the Science Research of CAUC (3122013k006) is highly appreciated.

\section{References}

[1] J. Li, C. E. Ekuma, I. Vekhter et al., "Physical properties of Ba2Mn2Sb2O single crystals," Physical Review B-Condensed Matter and Materials Physics, vol. 86, no. 19, Article ID 195142, 2012.

[2] C. Schlueter, P. Orgiani, T.-L. Lee et al., "Evidence of electronic band redistribution in $\mathrm{La}_{0.65} \mathrm{Sr}_{0.35} \mathrm{MnO}_{3-\delta}$ by hard $\mathrm{x}$-ray photoelectron spectroscopy," Physical Review B, vol. 86, Article ID 155102, 2012.

[3] B. F. Woodfield, M. L. Wilson, and J. M. Byers, "Lowtemperature specific heat of $\mathrm{La}_{1-x} \mathrm{Sr}_{x} \mathrm{MnO}_{3+\delta}$," Physical Review Letters, vol. 78, no. 16, pp. 3201-3204, 1997.

[4] M. R. Lees, O. A. Petrenko, G. Balakrishnan, and D. McK Paul, "Specific heat of $\operatorname{Pr}_{0.6}\left(\mathrm{Ca}_{1-x} \mathrm{Sr}_{x}\right)_{0.4} \mathrm{MnO}_{3}(0<\sim x<\sim 1)$," Physical Review B, vol. 59, no. 2, pp. 1298-1303, 1999.

[5] Z. Q. Li, E. Y. Jiang, D. X. Zhang et al., "Effect of annealing on polycrystalline $L a_{1-x} N a_{x} M n O_{z}$ ceramic," Physics Letters A, vol. 277, pp. 56-60, 2000.

[6] G. Reményi, M. Doerr, M. Loewenhaupt, S. Sahling, M. Saint-Paul, and P. Lejay, "Magnetostriction measurements at $\mathrm{Pr}_{0.65} \mathrm{Ca}_{0.35} \mathrm{MnO}_{3}$," Physica B: Condensed Matter, vol. 346-347, no. 1-4, pp. 83-86, 2004.

[7] M. Doerr, G. Reményi, M. Rotter et al., "Magnetoelastic investigations at $\operatorname{Pr}_{1-x} \mathrm{Ca}_{x} \mathrm{MnO}_{3}(x=0.35,0.5)$ manganites," Journal of Magnetism and Magnetic Materials, vol. 290-291, p. 906, 2005.

[8] L. Ghivelder, I. A. Castillo, and N. M. Alford, "Specific heat of $\mathrm{La}_{1-x} \mathrm{Ca}_{x} \mathrm{MnO}_{3-\delta}$," Journal of Magnetism and Magnetic Materials, vol. 189, pp. 274-282, 1998.

[9] V. N. Smolyaninova, A. Biswas, X. Zhang et al., "Anomalous field-dependent specific heat in charge-ordered $\operatorname{Pr}_{1-x} \mathrm{Ca}_{x} \mathrm{MnO}_{3}$ and $\mathrm{La}_{0.5} \mathrm{Ca}_{0.5} \mathrm{MnO}_{3}$," Physical Review $B$, vol. 62, Article ID R14303, 2000.

[10] L. Ghivelder, I. A. Castillo, M. A. Gusm o, J. A. Alonso, and L. F. Cohen, "Specific heat and magnetic order in $\mathrm{LaMnO}_{3+\delta}$ ", Physical Review B-Condensed Matter and Materials Physics, vol. 60, no. 17, pp. 12184-12190, 1999.

[11] J. E. Gordon, R. A. Fisher, Y. X. Jia et al., "Specific heat of $\mathrm{Nd}_{0.67} \mathrm{Sr}_{0.33} \mathrm{MnO}_{3}$," Physical Review B, vol. 59, no. 1, pp. 127-130, 1999. 
[12] P. Raychaudhuri, C. Mitra, A. Paramekanti, R. Pinto, A. K. Nigam, and S. K. Dhar, "The metal-insulator transition and ferromagnetism in the electron-doped layered manganites $\mathrm{La}_{2.3-x} \mathrm{Y}_{x} \mathrm{Ca}_{0.7} \mathrm{Mn}_{2} \mathrm{O}_{7} \quad(\mathrm{x}=0,0.3,0.5)$," Journal of Physics Condensed Matter, vol. 10, no. 12, pp. L191-L198, 1998.

[13] C. L. Lu, K. F. Wang, S. Dong, J. G. Wan, J.-M. Liu, and Z. F. Ren, "Specific heat anomalies and possible Griffiths-like phase in $\mathrm{La}_{0.4} \mathrm{Ca}_{0.6} \mathrm{MnO}_{3}$ nanoparticles," Journal of Applied Physics, vol. 103, no. 7, Article ID 07F714, 2008.

[14] A. Daoud-Aladine, J. Rodríguez-Carvajal, L. Pinsard-Gaudart, M. T. Fernández-Díaz, and A. Revcolevschi, "Zener polaron ordering in half-doped manganites," Physical Review Letters, vol. 89, Article ID 097205, 2002. 

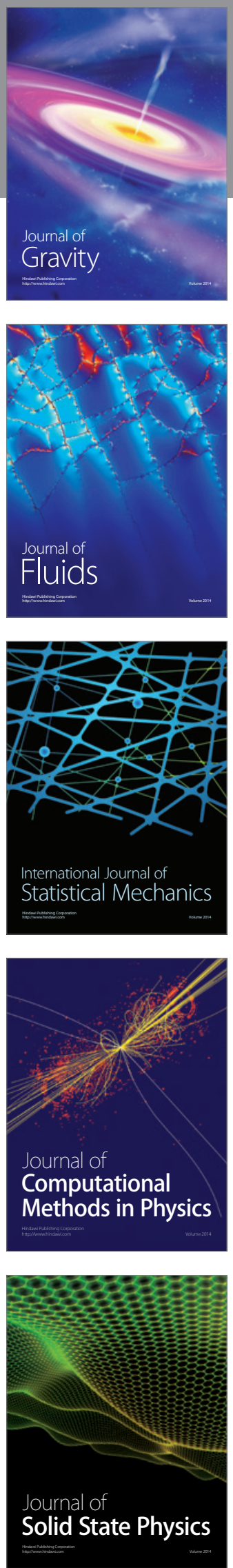

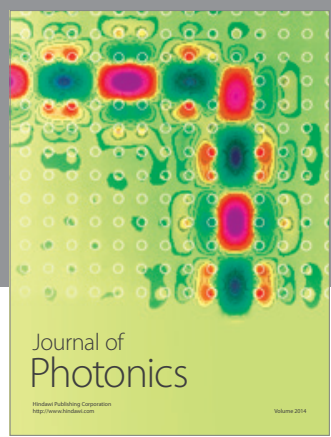

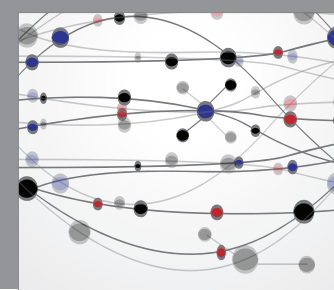

The Scientific World Journal

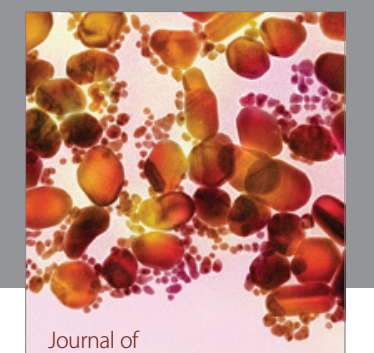

Soft Matter
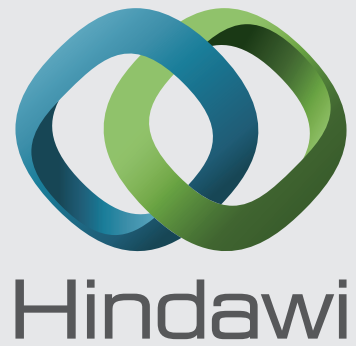

Submit your manuscripts at

http://www.hindawi.com
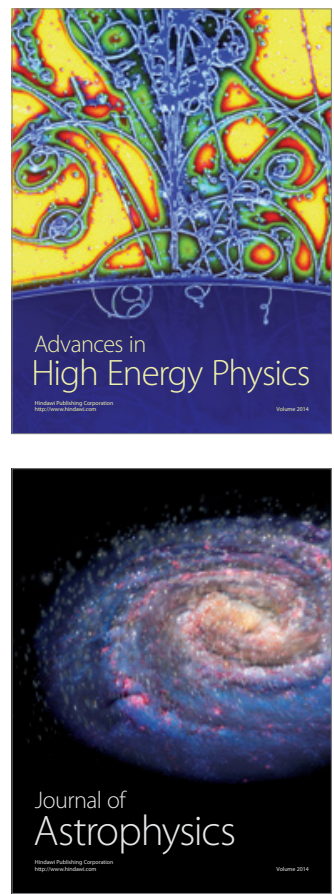
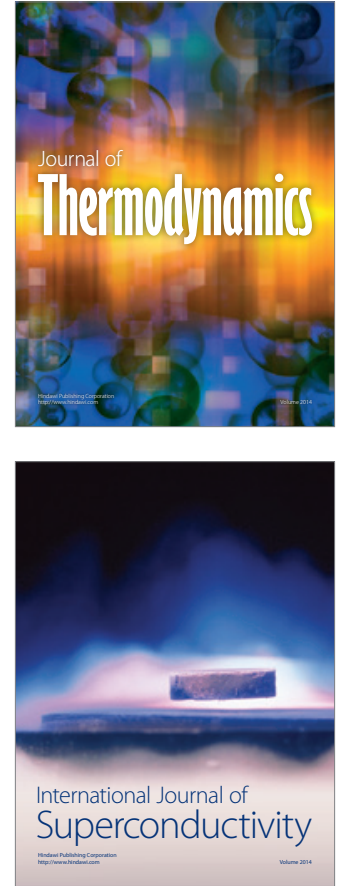
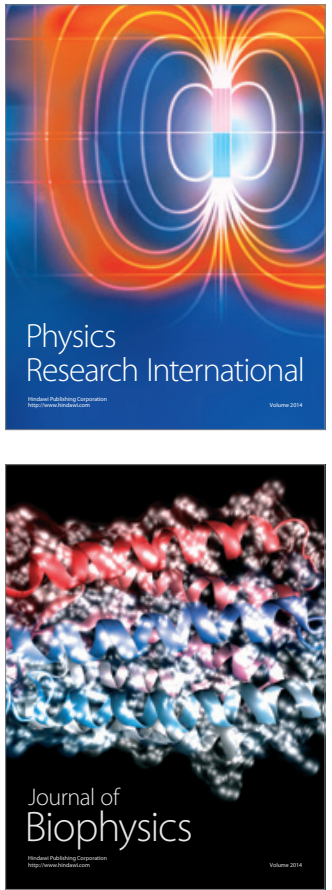
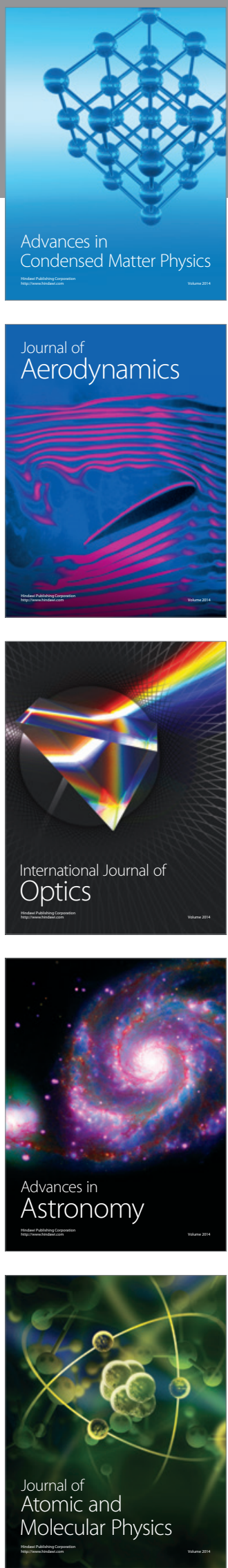